\title{
Direction of Umbilical Cord Twist and its Characteristics - a Pilot Study
}

Althea V. Pinto,' Alex X. Chakiath, ' Prudhvi Dasari,' Vilekith Reddy, ${ }^{1}$ Shirley George, ${ }^{1}$ Nachiket Shankar,'

\begin{abstract}
Background: A right-sided umbilical cord twist is associated with the presence of a single umbilical artery, congenital malformations and placenta praevia. Methods: It was an observational study. Data was collected from 137 umbilical cords, all from live births and their patient records. The gestational ages ranged from 28 weeks to 41 weeks. The umbilical cords were categorized into right or left, based on the direction of twist. The independent sample T test and the Chi square test were used to analyze the differences between groups. Results: The prevalence of left twist was $84 \%$. Right twist was significantly associated with a larger Hyrtl's anastomosis ( $p=0.029)$ and gestational diabetes $(\mathrm{p}=0.027)$. Conclusion: Two previously unreported associations with right twist of the umbilical cord, gestational diabetes and an increase in the diameter of Hyrt's anastomosis, were noted in the present study.
\end{abstract}

Keywords: Diabetes Gestational, Umbilical Cord, Embryonic Structures (Source: MeSH-NLM)

\section{Introduction}

The umbilical cord forms the only connection between the placenta and the developing fetus. It is surrounded by the amnion, and contains a pair of umbilical arteries and an umbilical vein supported by loose connective tissue, the Wharton's jelly. The umbilical vessels carry blood to and from the fetus and placenta, and thus, are critical to the survival of the fetus. A fully developed umbilical cord has an average length of $50 \mathrm{~cm}$, and is 1 to $2 \mathrm{~cm}$ in diameter. Close to the insertion of the cord on to the placenta the two umbilical arteries are usually connected by a transverse anastomosis called Hyrt's anastomosis (Figure 1). The umbilical cord is usually attached near the center of the placenta, from where the umbilical vessels ramify under the amnion and pass into the placenta. Valeen Pinto is currently doing her internship at St. John's Medical College Hospital, Bangalore, India.

The umbilical cord is usually twisted on itself, with the number of turns ranging from a few to over 300 (Figure 1)., ${ }^{1,2}$ The direction of this twist is usually left sided. ${ }^{3}$ Proposed reasons for this twisting include hemodynamic factors and fetal movements. ${ }^{3}$ Right sided twist is associated with the presence of a single umbilical artery (SUA), congenital malformations, placenta praevia and stillbirths. ${ }^{3-6}$ Previous studies from India have described the helical structure of the umbilical cord and documented the associations between the coiling index and adverse perinatal outcomes. Both hypo and hyper-coiling were noted to be associated with several adverse antenatal perinatal outcomes. ${ }^{7.8}$ The aim of the present study was to study the characteristics of umbilical cord twist direction among umbilical cords from South India.

\section{Methods}

The study was an observational study. The STROBE guidelines were used to conduct the study. Ethical clearance was obtai- ned from the institutional ethical review board. A total of 137 (131 singleton and 6 twins) umbilical cords were studied, all from live births. The gestational ages ranged from 28 weeks to 41 weeks. The placentae were collected from the labor room, tagged to enable identification and subsequently placed in a container with formalin. They were then transported to the department of anatomy for further analysis. During collection of the placentae, relevant details were noted from the case sheets of the patients.

There were two primary objectives of the present study. The first was to estimate the difference in the following numerical variables between umbilical cords with right and left twist: 1 ) Age of the mother; 2) Difference in diameters of umbilical artery; 3) Diameter of Hyrtl's anastomosis; 4) Birth weight of the neonate. The second objective was to compare the prevalence of the following categorical variables between umbilical cords with right and left twist: 1) Cender of the neonate; 2) Type of delivery; 3) Gestational diabetes; 4) Pregnancy induced hypertension.

In the anatomy department, the parameters mentioned in the objectives i.e. the twist of the umbilical cord, presence of SUA, presence and diameter of Hyrtl's anastomosis and diameters of the right and left umbilical arteries were noted. The umbilical cord twist was determined as shown in Figure $2 .{ }^{9}$ An incision was made on the amnion covering the umbilical cords, close to the site of their insertion onto the placentae. The umbilical arteries were dissected free from the surrounding Wharton's jelly and traced towards the fetal surface of the placenta. The Hyrtl's anastomosis was identified and dissected out from the surrounding connective tissue. No histological study was performed. All diameters were measured using digital Vernier 
Figure 1. A Dissection to show Hyrtl's Anastomosis between the Two Umbilical Arteries. FP - Fetal Surface of the Placenta; HA - Hyrtl's Anastomosis, UA - Umbilical Arteries.

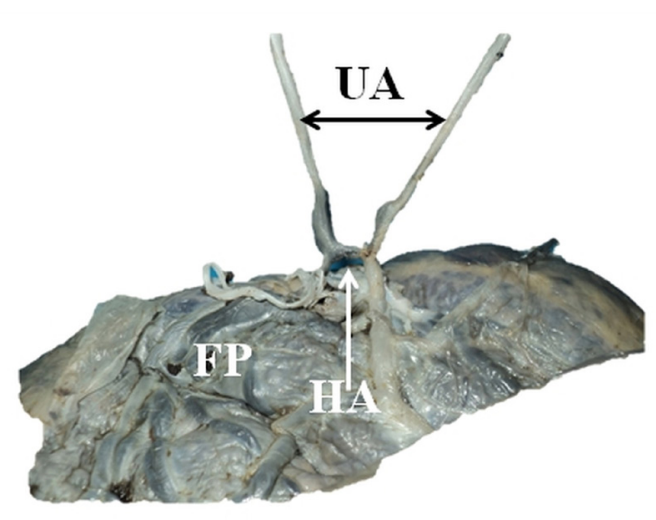

calipers. The independent sample $\mathrm{T}$ test and the Chi square test were used to analyze differences between groups with a significance level ( $p$ value) set at 0.05 . Statistical analysis was performed using SPSS version 16.

Figure 2. Determination of the Direction of Twist using the Limbs of the Capital Letter V. ${ }^{6}$ Umbilical Cords with Left Twist (LT) and Right Twist (RT) have Grooves of Coiling Parallel to the Respective Limbs of the V. FP - Fetal Surface of the Placenta UA - Umbilical Arteries

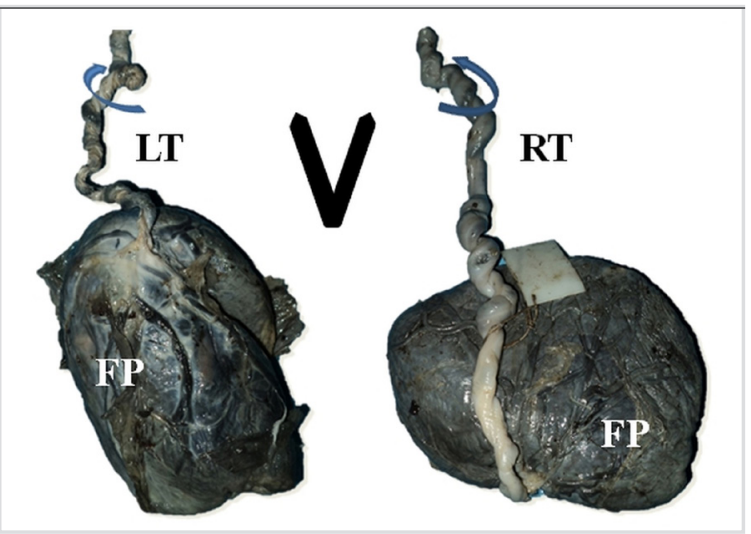

\section{Results}

The prevalence of left twist was $84 \%$ (117 of 137). A single Hyrtl's anastomosis was noted in all the placentae. The results regarding the numerical variables are summarized in Table 1. The diameter of Hyrtl's anastomosis was significantly higher in umbilical cords with a right twist $(3.4 \pm 1.7 \mathrm{~mm}$ versus $2.6 \pm 1.3$ $\mathrm{mm}, \mathrm{p}=0.029$ ). Among the categorical variables, no significant differences were noted with respect to gender, type of delivery and pregnancy induced hypertension. However, the prevalence of gestational diabetes was noted to be significantly higher in right twist (20\%) as compared to left twist ( $4.3 \%$ ) with a p value of 0.027 as noted in Table 1.

\section{Discussion}

The umbilical cord twist is an important feature of the umbilical cord. Evidence that the helical nature of the cord is not purely of genetic origin comes from the fact that there is an absence of concordance in monozygotic twins.? Also in intrauterine fetal deaths and in twins, this twist is less pronounced or absent, suggesting that fetal movements play a role. ${ }^{3}$ The absence of a twist should raise the suspicion of the presence of a congenital malformation and adverse foetal prognosis.,10 An interesting finding is that a left twist of the cord outnumbers the right twist in a ratio of $7: 1,3$ and the prevalence of right twist in the present study is similar. A right twist of the umbilical cord is more commonly associated with a SUA and placenta praevia than a left twist. ${ }^{3.5}$ The present study shows a significant difference in the proportion of mothers with gestational diabetes between umbilical cords with a right and left twist, suggesting the possibility of associations between them. Previously diabetes was shown to be associated with hypo or hyper-coiling of the umbilical cord. ${ }^{8,11}$ Another study showed that there were definite pathological changes in the umbilical arteries and Wharton's jelly in mothers with gestational diabetes. ${ }^{12}$ These effects on the umbilical cord may be a likely cause for the effects on the direction of coiling that were observed.

Hyrtl's anastomosis is the only connection between the two umbilical arteries. ${ }^{13}$ It is thought to play the role of either, a safety valve or a pressure stabilizer, between the umbilical arteries at the placental insertion. ${ }^{14}$ In a study conducted on 67 full term placentae from adequate for gestational age infants, Hyrtl's anastomosis was found to be absent in only 4 of the placentae. ${ }^{13}$ It has been found that the size of the anastomosis is related to the symmetry in the size of the umbilical arteries. Greater symmetry is associated with a smaller size, while asymmetry is associated with a larger size. ${ }^{13,15}$ In the present study, though the difference between the diameters of the umbilical arteries was more in right twist, this was not significant.

Table 1. Differences in the Numerical and Categorical Variables between the Groups with Left and Right Umbilical Cord Twist

\begin{tabular}{|c|c|c|c|}
\hline Variable & $\begin{array}{l}\text { Left twist } \\
\mathrm{n}=117\end{array}$ & $\begin{array}{c}\text { Right twist } \\
n=20\end{array}$ & $\mathrm{p}$ Value \\
\hline Age of mother (years) & $25.9 \pm 4.9$ & $27.1 \pm 3.6$ & 0.327 \\
\hline Diameter of Hyrtl's anastomosis (mm) & $2.6 \pm 1.3$ & $3.4 \pm 1.7$ & 0.029 \\
\hline Type of delivery (\% of Caesarean sections) & $39(33 \%)$ & $11(55 \%)$ & 0.067 \\
\hline Pregnancy induced hypertension ( $\%$ of positive diagnoses) & $20(17 \%)$ & $4(20 \%)$ & 0.765 \\
\hline Gestational diabetes ( $\%$ of positive diagnoses) & $5(4 \%)$ & $4(20 \%)$ & 0.027 \\
\hline
\end{tabular}


This greater asymmetry in the umbilical arteries with a right twist is a possible reason for the larger Hyrtl's anastomoses seen in these cases. A limitation of the present study was that only a few independent variables were considered. Other variables that could be considered include the following: umbilical cord insertion, distribution of chorionic plate vessels (magistral versus dispersed), evidence of umbilical cord knots and length of the umbilical cord. Future studies with larger sample sizes and more robust study designs such as case-control and cohort studies are required to confirm the findings of the present study.

\section{Conclusion}

Two previously unreported associations with right twist of the umbilical cord, gestational diabetes and increase in the diameter of Hyrtl's anastomosis, were noted in the present study. The association of a right-sided umbilical cord twist and gestational diabetes may be due to vascular changes that are produced as a result of the disease. Additionally, the relationship between asymmetry in the size of the umbilical arteries and the direction of twist of the umbilical cord needs to be explored in future studies.

\section{References}

1. Standring S, editor-in-chief. Gray's anatomy. 39th ed. Edinburgh: Elsevier Churchill Livingstone Publishers; 2006.

2. Edmonds HW. The spiral twist of the normal umbilical cord in twins and in singletons. Am J Obstet Gynecol. 1954;67(1):102-20.

3. Lacro RV, Jones KL, Benirschke K. The umbilical cord twist: origin, direction, and relevance. Am J Obstet Gynecol. 1987;157(4 Pt 1):833-8.

4. Abuhamad AZ, Shaffer W, Mari G, Copel JA, Hobbins JC, Evans AT. Single umbilical artery: does it matter which artery is missing? Am J Obstet Gynecol. 1995;173(3 Pt 1):728-32.

5. Kalish RB, Hunter T, Sharma G, Baergen RN. Clinical significance of umbilical cord twist. Am J Obstet Gynecol. 2003;189(3):736-9.

6. Ernst LM, Minturn L, Huang MH, Curry E, Su EJ. Gross patterns of umbilical cord coiling: correlations with placental histology and stillbirth. Placenta. 2013;34(7):583-8.

7. Chaurasia BD, Agarwal BM. Helical structure of the human umbilical cord. Acta Anat (Basel). 1979;103(2):226-30.

8. Chitra T, Sushanth YS, Raghavan S. Umbilical coiling index as a marker of perinatal outcome: an analytical study. Obstet Gynecol Int. 2012;2012:213689. 9. Finberg HJ. Avoiding ambiguity in the sonographic determination of the direction of umbilical cord twists. J Ultrasound Med.1992;11(5):185-7.

10. Ente G, Penzer PH. The umbilical cord: normal parameters. J R Soc Health. 1991;111(4):138-40.

11. Ezimokhai M, Rizk DE, Thomas L. Abnormal vascular coiling of the umbilical cord in gestational diabetes mellitus. Arch Physiol Biochem. 2001;109(3):209-14.

12. Singh SD. Gestational diabetes and its effect on the umbilical cord. Early Hum Dev. 1986;14(2):89-98.

13. Ullberg U, Sandstedt B, Lingman G. Hyrtl's anastomosis, the only connection between the two umbilical arteries. A study in full term placentas from AGA infants with normal umbilical artery blood flow. Acta Obstet Gynecol Scand. 2001;80(1):1-6.

14. Gordon Z, Eytan 0, Jaffa AJ, Elad D. Hemodynamic analysis of Hyrtl anastomosis in human placenta. Am J Physiol Regul Integr Comp Physiol. 2007;292(2):977-82.

15. Raio L, Chezzi F, di Naro E, Franchi M, Balestreri D, Dürig P et al. In-utero characterization of the blood flow in the Hyrtl anastomosis. Placenta. 2001;22(6):597-601.

\footnotetext{
Acknowledgments

The authors would like to acknowledge the attendants in the Department of Anatomy and the nursing staff in the Department of Obstetrics and Gynaecology for their assistance.
}

Conflict of Interest Statement ct Funding

The Authors have no funding, financial relationships or conflicts of interest to disclose.

Cite as:

Pinto AV, Chakiath AX, Dasari P, Reddy V, George S, Shankar N. Direction of Umbilical Cord Twist and its Characteristics - a Pilot Study. Int J Med Students. 2013:1(3):120-2. 